\title{
Facile and Scalable Preparation of Pure and Dense DNA Origami Solutions**
}

\author{
Evi Stahl, Thomas G. Martin, Florian Praetorius, and Hendrik Dietz*
}

\begin{abstract}
DNA has become a prime material for assembling complex three-dimensional objects that promise utility in various areas of application. However, achieving user-defined goals with DNA objects has been hampered by the difficulty to prepare them at arbitrary concentrations and in user-defined solution conditions. Here, we describe a method that solves this problem. The method is based on poly(ethylene glycol)induced depletion of species with high molecular weight. We demonstrate that our method is applicable to a wide spectrum of DNA shapes and that it achieves excellent recovery yields of target objects up to $97 \%$, while providing efficient separation from non-integrated DNA strands. DNA objects may be prepared at concentrations up to the limit of solubility, including the possibility for bringing DNA objects into a solid phase. Due to the fidelity and simplicity of our method we anticipate that it will help to catalyze the development of new types of applications that use self-assembled DNA objects.
\end{abstract}

In the quest for achieving precise control over the positioning of matter from the bottom-up, various strategies for creating feature-rich DNA objects of arbitrary shapes with molecular weights in the multiple-megadalton regime have been developed. ${ }^{[1-3]}$ Objects made using a particular approach known as DNA origami, ${ }^{[2 a]}$ in which many short DNA single-strands are designed to form double-helical DNA domains with a long

[*] E. Stahl, Dr. T. G. Martin, F. Praetorius, Prof. Dr. H. Dietz Physik Department, Walter Schottky Institute Technische Universität München

Am Coulombwall 4a, 85748 Garching (Germany)

E-mail: dietz@tum.de

Homepage: http://bionano.physik.tu-muenchen.de

[**] We thank Christian Wachauf for designing the 4- and 10-helix bundle, Fabian Kilchherr for designing the 18-helix bundle, Jonas Funke for designing the "hinge" object, Matthias Schickinger for designing the "flake", Philip Ketterer for designing the 100-helix bundle, and Dominik Renn for technical assistance. This work was supported by the Deutsche Forschungsgemeinschaft through the Excellence Cluster Center for Integrated Protein Science, Nano Initiative Munich, the Sonderforschungsbereich SFB863, and the TUM International Graduate School of Science and Engineering (IGSSE). Additional funding came from a European Research Council Starting Grant 256270 (to H.D.).

D) Sur Supporting information for this article is available on the WWW under http://dx.doi.org/10.1002/anie.201405991.

of (c) 2014 The Authors. Published by Wiley-VCH Verlag GmbH \& Co. $\mathrm{KGaA}$. This is an open access article under the terms of the Creative Commons Attribution Non-Commercial License, which permits use, distribution and reproduction in any medium, provided the original work is properly cited and is not used for commercial purposes. single-strand template, have already found use in applications such as single-molecule sensing,${ }^{[4]}$ plasmonics, ${ }^{[5]}$ and structural biology ${ }^{[6]}$ Other objects are being explored as potential drug delivery vehicles. ${ }^{[7]}$

However, more often than not application requirements are at odds with the restricted set of conditions at which productive synthesis of DNA objects can be performed. ${ }^{[8]}$ For instance, the concentration of objects is typically limited to the dilute nanomole-per-liter regime when using current methods of assembly and purification. ${ }^{[8 b, c]}$ Yet, many potential applications-whether in solution, in cell culture, or in organisms-do require much more concentrated solutions to achieve measurable effects. Furthermore, co-factors such as the calibrated amounts of cations that are required for the self-assembly reactions ${ }^{[2 \mathrm{~b}, 9]}$ may interfere in prospective applications. Hence, in order to reach utility, the restricted conditions at which productive self-assembly of DNA objects can be carried out must be uncoupled from those required for intended applications. Here, we solve this problem and describe a scalable method for purifying and dissolving repeatedly and efficiently megadalton-scale DNA objects in user-defined buffers at user-defined concentrations. We demonstrate the utility of our method with 19 different objects made using the DNA origami approach, but the method is likely to be generally applicable to any highmolecular-weight DNA object, including objects made using either the DNA origami ${ }^{[2 a]}$ or template-free DNA tile approach. ${ }^{[3]}$

Our method exploits the phenomenon of depletion of high-molecular-weight species by excluded volume effects that occur when adding poly(ethylene glycol) (PEG) polymers as a crowding agent to a solution. Precipitation by PEG crowding has been used for decades to purify and concentrate DNA plasmids ${ }^{[10]}$ and phage particles. ${ }^{[11]}$ In previous work with one particular type of DNA objects that resembled filamentous phage particles, ${ }^{[12]}$ PEG precipitation was employed to reduce large elution volumes after an ionexchange-based purification of the folded objects from nonintegrated DNA strands - a task for which the step involving PEG-induced sedimentation apparently lacked in fidelity. The procedure that we describe here builds on these important previous successes, but we have tested and refined the method significantly with respect to recovery yields, separation capability from non-integrated DNA strands, reproducibility, scalability, and applicability to DNA objects with arbitrary shapes. The procedure that we arrived at consists in simply adding one volume of a calibrated precipitation buffer, followed by centrifugation to pellet precipitates, and exchanging the buffer (Scheme S1). Tests in which we varied the composition of the precipitation buffer (type 
a)
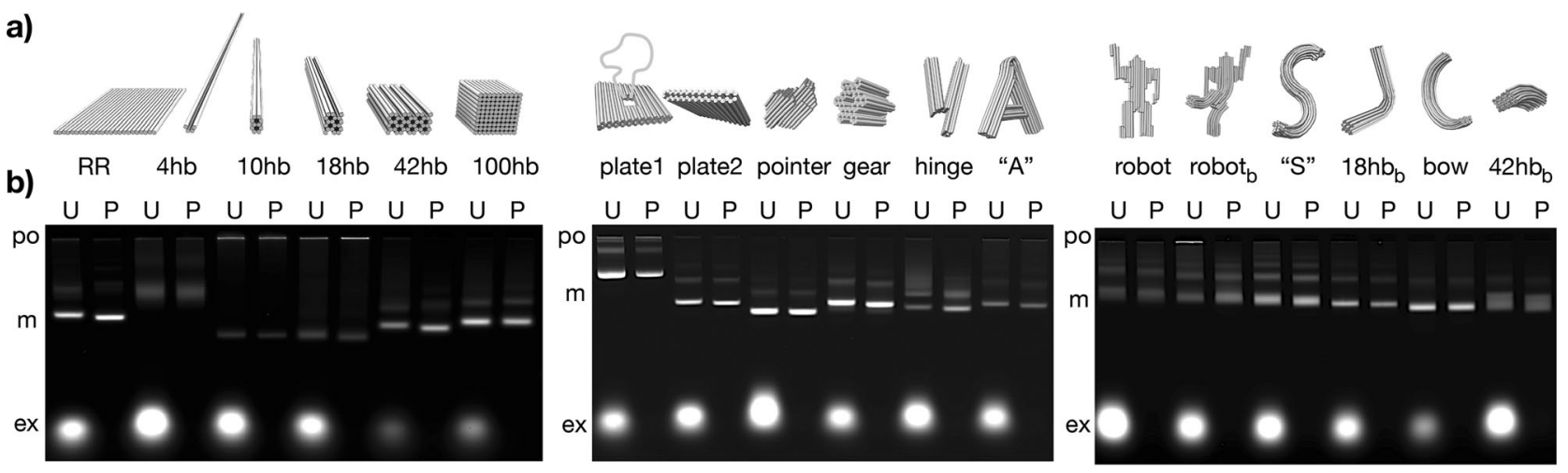

c)
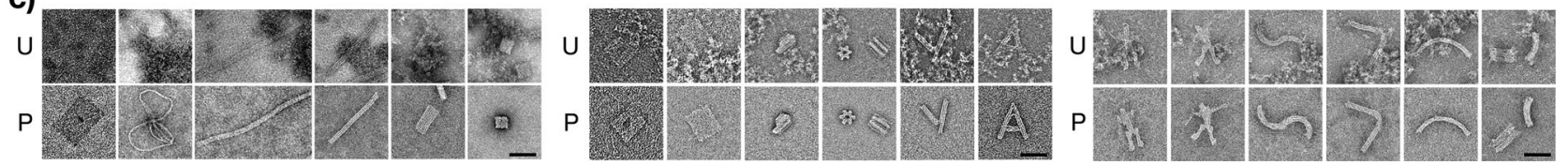

Figure 1. PEG purification of a library of DNA origami objects. a) Illustration (RR to $100 \mathrm{hb}$ ) and CanDo-computed ${ }^{[20]}$ models (platel to $42 \mathrm{hb}$ ) of a panel of DNA objects comprising a variant of Rothemund's single-layer DNA rectangle (RR) ${ }^{[2 a]}$ and five multilayer objects (four-, ten-, 18-, or 42helix bundle in honeycomb lattice design; 100 -helix bundle in square lattice design), ${ }^{\left[{ }^{9]}\right.}$ a plate-like object with aperture and double-stranded loop (plate 1), ${ }^{\left[{ }^{[c]}\right]}$ a plate-like object (plate2), ${ }^{\left[{ }^{[4]}\right.}$ an asymmetric 82 -helix bundle in square lattice design (pointer), ${ }^{[2 h]}$ a gear-like object (gear), ${ }^{\left[{ }^{[18]}\right.}$ a flexible hinged-bar object (hinge), ${ }^{[18]}$ a letter-A-like object ("A"), ${ }^{[206]}$ a straight and bent version of a robot-like object (robot, robot $\left.{ }_{b}\right),{ }^{\left[{ }^{[20]}\right.}$ a letter-S-like object $\left(" \mathrm{~S}\right.$ "), ${ }^{[20 b]}$ a bow-like object (bow), ${ }^{[20 b]}$ and bent versions of an 18 -helix bundle and a 42 -helix bundle $\left(18 \mathrm{hb} b_{b}, 42 \mathrm{hb} b\right)$, ${ }^{[2,18]}$ The library of objects thus samples a wide spectrum of shapes, aspect ratios, and mechanical properties. b) Images of agarose gels on which unpurified self-assembly reaction mixtures $(U)$ and PEG purified samples $(P)$ of the objects listed above in (a) were electrophoretically separated. Labels: po, gel loading pocket; $m$, properly folded monomers; ex, non-integrated excess staple strands. c) Exemplary TEM micrographs of single particles in unpurified reaction mixtures $(U)$ and PEG-purified samples $(P)$. Scale bars: $50 \mathrm{~nm}$.

and concentration of depletant, type and concentration of cations), the time of incubation in the precipitation buffer, and details of the pelleting procedure helped us converge on conditions that give simultaneously excellent recovery of the high-molecular-weight target species and good separation from non-integrated DNA oligonucleotides (Note S3).

First, we demonstrate that our method can be used for purifying and concentrating a broad range of DNA objects with various aspect ratios and mechanical properties. To this end, we have self-assembled and purified a library of different DNA origami objects (Figure 1). Unpurified and purified samples were analyzed using agarose gel electrophoresis and direct imaging by transmission electron microscopy (TEM; Figure $1 b, c)$. Using our method, all of the objects that we tested could be purified satisfactorily from non-integrated low-molecular-weight DNA oligonucleotides (Figure 1b). Some of the objects that we tested contained by design polythymidine tails at the helical interfaces (10- and 100-helix bundle, plate-like objects, and pointer object), whereas all other objects had single-stranded scaffold DNA loops with various sequences at the helical interfaces. The loops and the tails both served the purpose of preventing aggregation by blunt-end association. After PEG purification, the samples with polythymidine tails exhibited the same electrophoretic mobility as before the treatment. By contrast, the samples with single-stranded scaffold loops had greater mobility after PEG purification than before. We attribute the enhanced mobility to the removal of excess DNA oligonucleotides that stick to the single-stranded loops in the unpurified samples. When we added excess DNA oligonucleotides to a PEG- purified object with single-stranded DNA loops, it migrated again with the reduced mobility as observed before PEG purification (Figure S6b).

To test the efficacy of our method with respect to recovery yield and purity of the target species, we performed ten successive cycles of precipitating and redissolving one particular multilayer DNA origami object (the 42-helix bundle, Figure 1, lane 5). A gel-electrophoretic analysis of samples taken after each cycle (Figure $2 \mathrm{a}-\mathrm{c}$ ) indicated an average recovery efficiency of $97 \pm 5 \%$ per cycle $(\mathrm{N}=30)$. One cycle of PEG purification removed $96 \%$ of non-integrated staple strands. Two cycles removed $99.4 \%$ of all excess staples, and three cycles gave samples that were essentially free of nonintegrated staple strands (Figure $2 b, c$ ).

Similarly to PEG-based purification, molecular weight cut-off filtration also allows for separating non-integrated DNA staple strands from high-molecular-weight self-assembly products. ${ }^{[13]}$ To compare the performance of PEG purification versus filtration, we subjected test reaction mixtures to one and five rounds of PEG-based purification and filtration, respectively (Figure $2 \mathrm{~d}-\mathrm{f}$ ). According to a gelelectrophoretic analysis of the samples after the procedure, we recovered $53 \%$ of the sample after one cycle of filtration, and $49 \%$ after five cycles. We attribute the significant losses incurred in particular during the first cycle of filtration to material that adhered irreversibly to the filtration membrane. By contrast, after one cycle of PEG purification we recovered $93 \%$ of the sample in this experiment, and $84 \%$ after five cycles. Filtration was also less efficient than PEG purification with respect to staple strand removal: twice as many residual 
a)

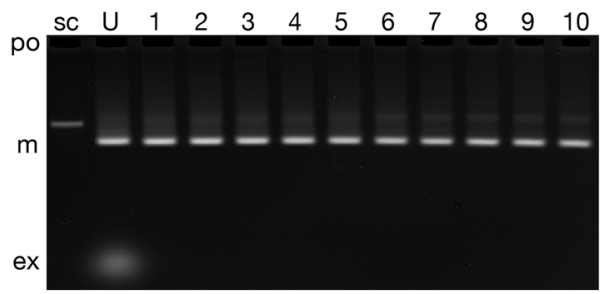

b)

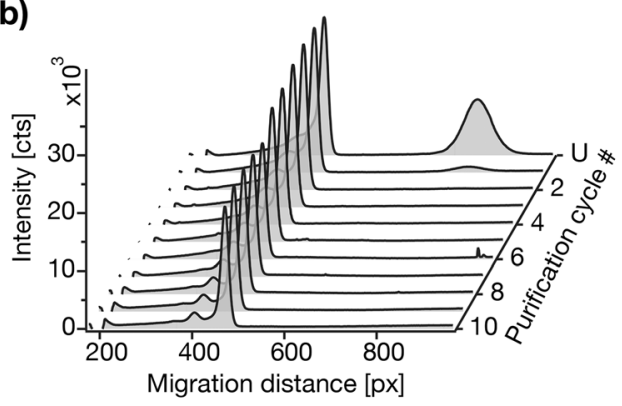

c)

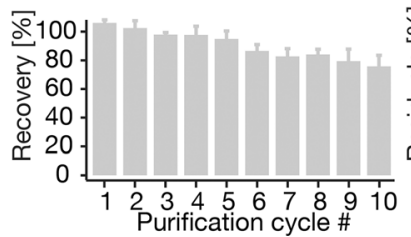

d)

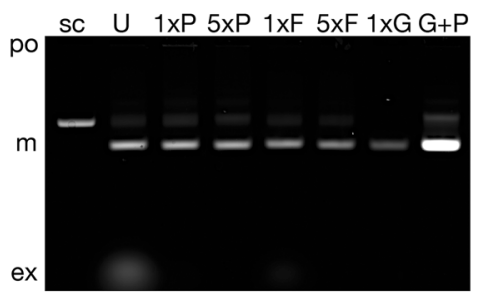

e)

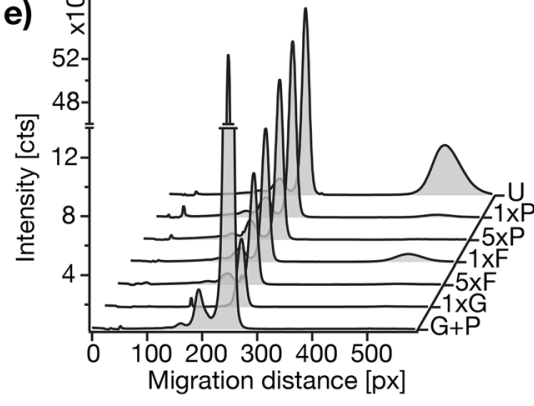

f)

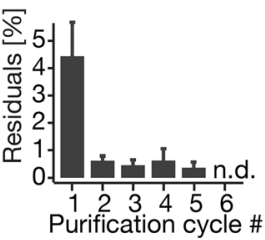

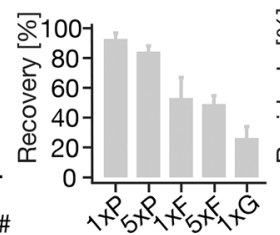

g)

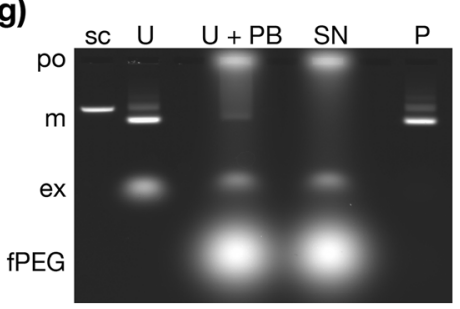

h)

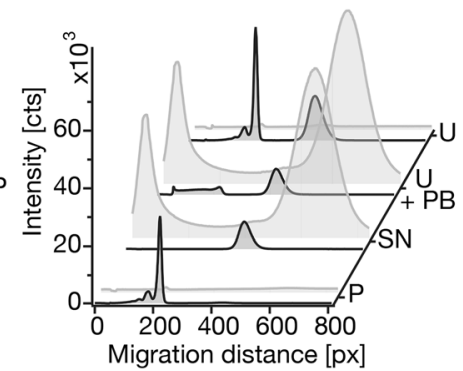

i)

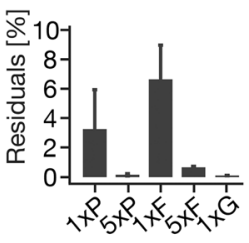

\begin{tabular}{r|c|c|c|c}
$c[\mathrm{M}]$ & $\mathrm{U}$ & $\mathrm{PB}$ & $\mathrm{U}+\mathrm{PB}$ & $\mathrm{P}$ \\
\hline PEG & -- & $1.8 \cdot 10^{-2}$ & $9 \cdot 10^{-3}$ & $5.4 \cdot 10^{-5}$ \\
dsDNA & $5 \cdot 10^{-8}$ & -- & $2.5 \cdot 10^{-8}$ & $\sim 5 \cdot 10^{-8}$ \\
\hline \multicolumn{4}{r}{ residual PEG [\%] } & $\sim 0.3$
\end{tabular}

Figure 2. Agarose-gel-electrophoretic characterization of PEG purification using exemplarily a 42-helix bundle object. a) Image of a gel on which samples extracted after each of ten consecutive PEG purification cycles (1-10) were separated. Labels: sc, reference sample containing only scaffold strands; $U$, unpurified self-assembly reaction mixture; po, gel loading pocket; $m$, folded objects; ex, non-integrated excess staple strands. b) Cross-sectional lane profiles determined from (a). c) Recovery of folded objects (left) and residuals of excess staple strands (right) relative to unpurified reaction mixture, as determined by integrating and comparing the areas of the peaks reflecting folded objects and excess strands, respectively. The experiment was run in triplicate, each experiment gave data as in (a). Error bars in (c) indicate the standard deviation in the recovery and residuals, respectively. d) Image of a gel containing samples extracted after one and five cycles of PEG purification (1xP, 5xP), after one and five cycles of molecular-weight cut-off filtration (1xF, 5xF), after AGE extraction (1xG), and after PEG purification of a previously AGEextracted sample $(G+P)$. $U$ refers to the unpurified self-assembly reaction mixture. Other labels as in (a). e) Cross-sectional lane profiles determined from (d). f) Recovery of folded objects and residual excess strands as in (c) but for samples as in (d). Values were obtained from three independent experiments, each giving data as in (d). Error bars indicate the standard deviation in the recovery and residuals, respectively. g-i) Estimation of residual PEG content in purified samples using fluorescein-labeled PEG (fPEG). g) Overlay image of two scans of the same gel, recorded separately for the ethidium bromide and fluorescein emission channels. Samples were taken before $(U)$ and after $(U+P B)$ addition of precipitation buffer $(\mathrm{PB})$ and compared to the supernatant $(\mathrm{SN})$ and the redissolved pellet $(\mathrm{P})$ of the precipitation. h) Cross-sectional lane profiles from ethidium bromide channel (dark gray) and fluorescein channel (light gray). i) Estimated concentrations of folded DNA objects (dsDNA) and PEG at all steps of a PEG purification cycle. Labels: fPEG, fluorescein-labeled PEG; other labels as in (a).

staple strands were present in the once-filtered sample as compared to a once-PEG-precipitated sample. Five cycles of filtration were necessary to remove more than $99.3 \%$ of residual staple strands (Figure $2 \mathrm{e}, \mathrm{f}$ ).

Agarose gel electrophoresis (AGE) is a popular method for purifying selected species with a particular electrophoretic mobility by band excision and physical extraction from the gel matrix. ${ }^{[14]}$ AGE extraction can thus be employed to remove non-integrated DNA oligonucleotides as well as species of greater molecular weight such as aggregates that might have been produced during molecular self-assembly reactions or postprocessing steps. Two important drawbacks of AGE extraction, however, are notoriously low recovery yields (Figure $2 \mathrm{~d}-\mathrm{f})^{[8 \mathrm{~b}, \mathrm{c}]}$ and the fact that the procedure results in dilute solutions containing the purified species at concentrations in the low nanomolar range. Combining AGE extraction with our PEG purification method, however, provides a convenient route for preparing pure target species at essentially any desired concentration, which we illustrate here exemplarily by first AGE-purifying a multilayer DNA origami object from all other species in the self-assembly reaction mixture, and then concentrating this sample tenfold (Figure 2d-f).

Our method relies on adding PEG polymers as a precipitating agent. To quantify exemplarily the extent of residual PEG polymers in a purified sample with a particular scale, we prepared a precipitation buffer that contained a fraction of PEG polymers labeled with fluorescein isothiocyanate (FITC) and used it to precipitate a multilayer DNA origami object (the 42-helix bundle). The pelleted precipitate was redissolved in PEG-free buffer in the starting volume. Agarose gel electrophoresis of the thus treated sample and laser scanning of the gel allowed us to quantify the remainder of FITC-labeled PEG polymers in the purified sample 
(Figure $2 \mathrm{~g}, \mathrm{~h}$ ). Our analysis shows that $99.7 \%$ of the FITClabeled PEG molecules were removed. We consider this as an estimate for the overall rate of removal of PEG molecules (Figure 2i). However, if desired, residual PEG can be removed completely from the PEG-purified and concentration-enhanced sample using low-molecular-weight filtration membranes (Note S4).

Our refined PEG purification method is scalable and free from restrictions with respect to the volumes and compositions of the starting sample and the target sample. To illustrate these properties, we have self-assembled one single-layer and two multilayer DNA origami test objects at a large $20 \mathrm{~mL}$ scale (as opposed to the typical $100 \mu \mathrm{L}$ scale reaction mixtures). The single-layer object was a variant of Rothemund's rectangle (Figure 1, lane 1). One multilayer object was the 42-helix bundle introduced above, but modified with a single fluorescent Atto655 dye. The other multilayer object was a 24-helix bundle in honeycomb-packing that was labeled with, on the average, ten fluorescent cyanine-3 dye molecules distributed over 30 single-stranded sites per object. We precipitated the high-molecular-weight reaction products, removed the supernatant that contained non-integrated DNA oligonucleotides and then dried the precipitates to obtain several milligrams of each object within solid material (Figure $3 \mathrm{a}-\mathrm{c}$ ). We attribute about $50 \%$ of the measured dry weight to PEG molecules, salts, and residual water (Note S5).

To test whether the objects survive drying, we redissolved the solid material in buffer and analyzed the thus obtained solutions with TEM and gel electrophoresis. TEM micro- a)

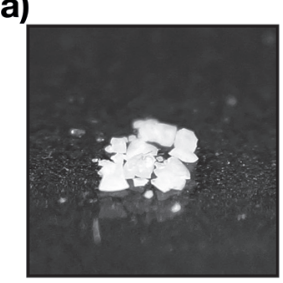

e)

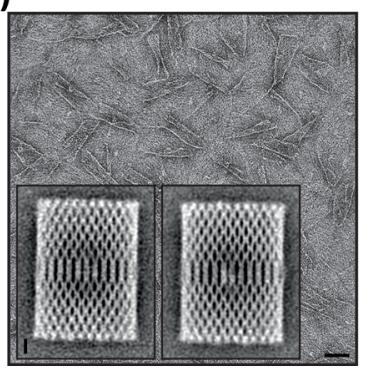

b)

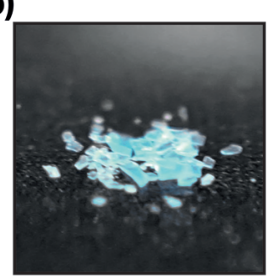

f)

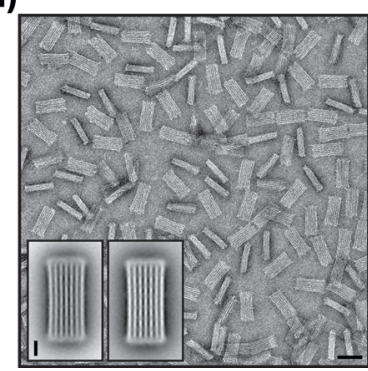

c)

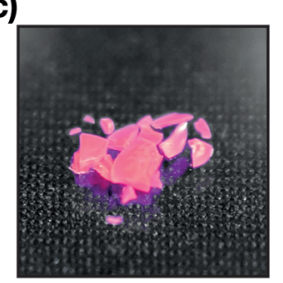

g)

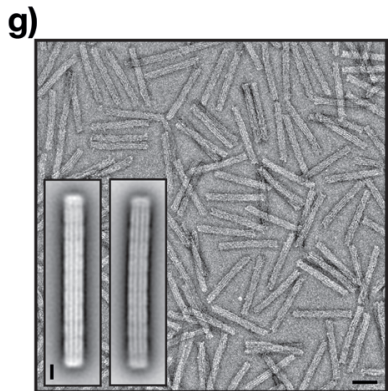

Figure 3. Preparation of solid material and dense solutions containing intact DNA objects. a) Approximately $6.8 \mathrm{mg}$ of solid material containing a variant of Rothemund's rectangle (unlabeled). b) Approximately $5.5 \mathrm{mg}$ of solid material containing 42-helix bundle objects, labeled each with one ATTO655 dye. c) Approximately $8.4 \mathrm{mg}$ of solid material containing 24-helix bundle objects, labeled each with ten cyanine-3 dyes on average. d) Left: unpurified self-assembly reaction mixture containing approximately $50 \mathrm{nM}$ of folded 24-helix bundle objects and $150 \mathrm{nM}$ excess staple strands; center: dense solution containing approximately $5.6 \mu \mathrm{M}$ of folded 24 -helix bundle objects, prepared by redissolving the materials from (c); right: reference sample containing $70 \mu \mathrm{M}$ of a cyanine-3 modified DNA oligonucleotide. e-g) TEM micrographs of dried and redissolved single-layer rectangle (e), 42 helix bundle $(\mathrm{f})$, and 24-helix bundle $(\mathrm{g})$; insets: average single-particle micrographs obtained from non-dried (left) and dried and redissolved samples (right). Scale bars: $50 \mathrm{~nm}$ (field-of-view micrographs), $10 \mathrm{~nm}$ (insets). graphs revealed well-folded particles that were indistinguishable both from unpurified and once precipitated, but not dried, particles (Figure $3 \mathrm{e}-\mathrm{g}$ ). To evaluate the structural in the average micrographs obtained from dried versus mobility of Rothemund's rectangle, 42-helix bundle and 24 helix bundle particles remained unaffected by the precipitation, drying, and redissolving treatment (Figures S8 and S9). namples of 24-helix bundle using a fluorescent comparable for dried and non-dried samples which suggests objects (Figure S9). Taken together, the data indicates that single-layer and multilayer DNA origami objects do survive the precipitation and drying treatment without taking amage. the limits of solubility of the object. We redissolved the dried material to prepare concentrated solutions containing up to . 5.6 $\mu \mathrm{M}$ of the DNA objects (as measured by absorption spectroscopy), as opposed to the low $0.05 \mu \mathrm{M}$ concentration at which the objects were self-assembled. The average interparticle distance at $5 \mu \mathrm{M}$ concentration is on the order of $70 \mathrm{~nm}$, which is comparable to the absolute dimensions of the objects. The solutions were slightly turbid suggesting that the limit of solubility was reached.

The experiment performed here with the three objects illustrates how our method uncouples the highly restricted conditions at which productive self-assembly of DNA origami objects can be carried out from those required for downstream applications. For example, at the elevated concentrations that we prepared, the main functionality of the 24-helix bundle object, which is to carry fluorescent labels, can now be readily observed in solution by eye due to the intense color of the solution (Figure $3 \mathrm{~d}$, center). By contrast, at the initial self-assembly reaction conditions, the solutions were colorless (Figure $3 \mathrm{~d}$, left). The perception of color is due to the wavelengthspecific absorption of transmitted and reflected light and depends on the concentration of absorbing par- 
ticles in solution. Here, color may thus serve as a placeholder for other desirable effects that depend on particle concentration. Preparing sufficiently dense solutions so that macroscopic properties result from the features of designed nanoparticles is standard in other areas of nanotechnology, ${ }^{[16]}$ but has so far been quite difficult to achieve when dealing with self-assembled DNA objects.

To conclude, we showed that PEG purification is a versatile method for efficiently purifying and concentrating a broad range of DNA origami objects with various aspect ratios and mechanical properties. Combining AGE extraction with our PEG purification method enables preparing pure objects at essentially any desired concentration, albeit with material losses that are caused by AGE. In our experience, PEG purification by itself does not enhance (and also does not reduce) the tendency for aggregation of a given object at a given solution condition (see Figure 1). Recent advances in design strategies ${ }^{[9,17]}$ and synthesis methods ${ }^{[18]}$ support the high-yield self-assembly of desired objects with a significantly reduced formation of byproducts, which diminishes greatly the need for laborious and inefficient molecular-weightspecific purification such as AGE. For example, we designed the 42-helix bundle and the 100-helix bundle using improved strand-breaking and self-assembly procedures. These objects, and also the variant of Rothemund's single-layer DNA rectangle, the plates, and the pointer self-assembled with close to $100 \%$ yield (Figure 1). In such cases, our refined PEG purification method is an ideal choice for the high-yield preparation of pure samples that can be directly used in downstream applications.

Since our method allows preparing samples routinely at arbitrary concentrations, we anticipate that it will help to catalyze the development of new types of applications that use self-assembled DNA objects. For example, crystallization assays, protein-DNA interaction assays, and cell-culturebased screens for immune response or cytotoxicity typically all require micromolar concentrations of the active agent, which we can now prepare. Because PEG purification also allows exchanging the solvent repeatedly without incurring significant sample losses, it opens a route for using DNA objects as solid support platforms for chemical synthesis.

\section{Experimental Section}

PEG precipitation: Self-assembly reaction mixtures at $20 \mathrm{~mm} \mathrm{MgCl}_{2}$ were mixed $1: 1(\mathrm{v} / \mathrm{v})$ with precipitation buffer containing $15 \%$ PEG 8000 (w/v) (Ph.Eur.), 5 mm Tris, 1 mm EDTA, and $505 \mathrm{~mm} \mathrm{NaCl}$ (all chemicals from Carl Roth, Karlsruhe, Germany). The solution was mixed by tube inversion and spinned at $16000 \mathrm{~g}$, at room temperature (RT) for $25 \mathrm{~min}$ using a microcentrifuge (Eppendorf 5420, Hamburg, Germany). The supernatant was removed using a pipette. The pellet was dissolved in target buffer as indicated for each set of experiments and incubated for approximately $20 \mathrm{~h}$ at RT or $30^{\circ} \mathrm{C}$.

DNA object self-assembly: Structures were designed using caDNAno v0.2. ${ }^{[19]}$ DNA scaffold strands of $7249,7560,7704$, and 8064 bases length derived from the genome of bacteriophage M13 were used for assembly reactions. ${ }^{[2 b]}$ Staple oligonucleotide strands were prepared by solid-phase chemical synthesis (Eurofins MWG, Ebersberg, Germany, HPSF grade). Production of DNA objects was accomplished in one-pot reaction mixtures containing scaffold DNA at a concentration of $20 \mathrm{~nm}$ (default) or $50 \mathrm{~nm}$ (pointer in Figure S1, RR in Figures 3 and S8, $24 \mathrm{hb}, 42 \mathrm{hb}, 100 \mathrm{hb}$ ), staple DNA oligonucleotides at $200 \mathrm{~nm}$ each, and $5 \mathrm{~mm}$ TRIS, $1 \mathrm{~mm}$ EDTA, $20 \mathrm{~mm} \mathrm{MgCl}_{2}$, and $5 \mathrm{~mm} \mathrm{NaCl}(\mathrm{pH} 8)$. The reaction mixtures were subjected to a thermal annealing protocol using TETRAD (Biorad) thermal cycling devices. The mixtures were first incubated at $65^{\circ} \mathrm{C}$ for $15 \mathrm{~min}$ and then annealed from 60 to $40^{\circ} \mathrm{C}$ in steps of $1^{\circ} \mathrm{C}$ per $2-3 \mathrm{~h}$. The reaction products were stored at RT.

Agarose gel electrophoresis: Electrophoresis of the folded DNA objects was carried out in $2 \%$ agarose gels containing electrophoresis buffer ( $1 \mathrm{~mm}$ EDTA, $44.5 \mathrm{~mm}$ Tris base, $44.5 \mathrm{~mm}$ boric acid, and $11 \mathrm{~mm} \mathrm{MgCl}$, pH 8.4). The samples were electrophoresed for two hours at 70-90 V in a water-cooled gel box filled with electrophoresis buffer. The gels typically contained ethidium bromide at a concentration of $1 \mu \mathrm{M}$. The agarose gels were scanned using a Typhoon 9500 FLA laser scanner (GE Healthcare) at a resolution of $50 \mu \mathrm{m} / \mathrm{px}$ (ethidium bromide: excitation at $535 \mathrm{~nm}$, emission $>575 \mathrm{~nm}$; fluorescein: excitation at $473 \mathrm{~nm}$, emission $520-540 \mathrm{~nm}$ ) to give 16-bit tif image files, which we analyzed using ImageJ64 V1.47 (U.S. National Institutes of Health). Cross-sectional lane intensity profiles were computed by averaging over grayscale values within a $20-75$ pixel wide box drawn over the lane of interest. After linear background correction, the regions of interest were quantified by integrating the area under the peaks. Yields were estimated by comparing the intensity of bands of interest for treated versus untreated samples.

Negative-staining TEM: Samples were adsorbed on glow-discharged formvar-supported carbon-coated Cu400 TEM grids (Science Services, Munich) and stained using a $2 \%$ aqueous uranyl formate solution containing $25 \mathrm{~mm}$ sodium hydroxide. Imaging was performed using a Philips CM100 EM operated at $100 \mathrm{kV}$. Images were acquired using an AMT 4 Megapixel CCD camera. Micrograph scale bars were calibrated by imaging 2D catalase crystals and using the lattice constants as length reference. Imaging was performed at $\times 28500$ magnification.

Received: June 6, 2014

Revised: August 29, 2014

Published online: October 24, 2014

Keywords: DNA nanotechnology · DNA origami .

molecular crowding · poly(ethylene glycol) · self-assembly

[1] a) N. C. Seeman, Annu. Rev. Biochem. 2010, 79, 65-87; b) V. Linko, H. Dietz, Curr. Opin. Biotechnol. 2013, 24, 555-561.

[2] a) P. W. Rothemund, Nature 2006, 440, 297-302; b) S. M. Douglas, H. Dietz, T. Liedl, B. Hogberg, F. Graf, W. M. Shih, Nature 2009, 459, 414-418; c) H. Dietz, S. M. Douglas, W. M. Shih, Science 2009, 325, 725-730; d) Y. Ke, S. M. Douglas, M. Liu, J. Sharma, A. Cheng, A. Leung, Y. Liu, W. M. Shih, H. Yan, J. Am. Chem. Soc. 2009, 131, $15903-15908$; e) D. Han, S. Pal, J. Nangreave, Z. Deng, Y. Liu, H. Yan, Science 2011, 332, 342-346; f) E. S. Andersen, M. Dong, M. M. Nielsen, K. Jahn, R. Subramani, W. Mamdouh, M. M. Golas, B. Sander, H. Stark, C. L. Oliveira, J. S. Pedersen, V. Birkedal, F. Besenbacher, K. V. Gothelf, J. Kjems, Nature 2009, 459, 73-76; g) Y. Ke, N. V. Voigt, K. V. Gothelf, W. M. Shih, J. Am. Chem. Soc. 2012, 134, $1770-$ 1774; h) X. C. Bai, T. G. Martin, S. H. Scheres, H. Dietz, Proc. Natl. Acad. Sci. USA 2012, 109, 20012-20017.

[3] a) B. Wei, M. Dai, P. Yin, Nature 2012, 485, 623-626; b) Y. Ke, L. L. Ong, W. M. Shih, P. Yin, Science 2012, 338, 1177-1183.

[4] a) N. D. Derr, B. S. Goodman, R. Jungmann, A. E. Leschziner, W. M. Shih, S. L. Reck-Peterson, Science 2012, 338, 662-665; b) E. Pfitzner, C. Wachauf, F. Kilchherr, B. Pelz, W. M. Shih, M. Rief, H. Dietz, Angew. Chem. Int. Ed. 2013, 52, 7766-7771; Angew. Chem. 2013, 125, 7920-7925; c) R. Wei, T. G. Martin, U. Rant, H. Dietz, Angew. Chem. Int. Ed. 2012, 51, 4864-4867; 


\section{Angewandte}

Angew. Chem. 2012, 124, 4948-4951; d) M. Langecker, V. Arnaut, T. G. Martin, J. List, S. Renner, M. Mayer, H. Dietz, F. C. Simmel, Science 2012, 338, 932-936.

[5] A. Kuzyk, R. Schreiber, Z. Fan, G. Pardatscher, E. M. Roller, A. Hogele, F. C. Simmel, A. O. Govorov, T. Liedl, Nature 2012, 483, $311-314$.

[6] a) S. M. Douglas, J. J. Chou, W. M. Shih, Proc. Natl. Acad. Sci. USA 2007, 104, 6644-6648; b) M. J. Berardi, W. M. Shih, S. C. Harrison, J. J. Chou, Nature 2011, 476, 109-113; c) D. N. Selmi, R. J. Adamson, H. Attrill, A. D. Goddard, R. J. Gilbert, A. Watts, A. J. Turberfield, Nano Lett. 2011, 11, 657-660.

[7] a) S. M. Douglas, I. Bachelet, G. M. Church, Science 2012, 335, $831-834$; b) S. D. Perrault, W. M. Shih, ACS Nano 2014, 8, $5132-5140$.

[8] a) A. V. Pinheiro, D. Han, W. M. Shih, H. Yan, Nat. Nanotechnol. 2011, 6, 763-772; b) C. Lin, S. D. Perrault, M. Kwak, F. Graf, W. M. Shih, Nucleic Acids Res. 2013, 41, e40; c) G. Bellot, M. A McClintock, C. Lin, W. M. Shih, Nat. Methods 2011, 8, 192-194.

[9] T. G. Martin, H. Dietz, Nat. Commun. 2012, 3, 1103.

[10] G. O. Humphreys, G. A. Willshaw, E. S. Anderson, Biochim Biophys. Acta Nucleic Acids Protein Synth. 1975, 383, 457-463.

[11] a) K. R. Yamamoto, B. M. Alberts, R. Benzinger, L. Lawhorne, G. Treiber, Virology 1970, 40, 734-744; b) T. Gibaud, E. Barry, M. J. Zakhary, M. Henglin, A. Ward, Y. Yang, C. Berciu, R.
Oldenbourg, M. F. Hagan, D. Nicastro, R. B. Meyer, Z. Dogic, Nature 2012, 481, 348-351.

[12] G. Bellot, M. A. McClintock, J. J. Chou, W. M. Shih, Nat. Protoc. 2013, $8,755-770$.

[13] C. Steinhauer, R. Jungmann, T. L. Sobey, F. C. Simmel, P. Tinnefeld, Angew. Chem. Int. Ed. 2009, 48, 8870-8873; Angew. Chem. 2009, 121, 9030-9034.

[14] R. W. Thuring, J. P. Sanders, P. Borst, Anal. Biochem. 1975, 66, $213-220$

[15] K. F. Wagenbauer, C. H. Wachauf, H. Dietz, Nat. Commun. 2014, 5,3691

[16] E. Boisselier, D. Astruc, Chem. Soc. Rev. 2009, 38, 1759-1782.

[17] Y. Ke, G. Bellot, N. V. Voigt, E. Fradkov, W. M. Shih, Chem. Sci. 2012, 3, 2587-2597.

[18] J. P. Sobczak, T. G. Martin, T. Gerling, H. Dietz, Science 2012, $338,1458-1461$.

[19] S. M. Douglas, A. H. Marblestone, S. Teerapittayanon, A. Vazquez, G. M. Church, W. M. Shih, Nucleic Acids Res. 2009, $37,5001-5006$.

[20] a) C. Castro, F. Kilchherr, D. Kim, E. Shiao, T. Wauer, P. Wortmann, M. Bathe, H. Dietz, Nat. Methods 2011, 8, 221; b) D. Kim, F. Kilchherr, H. Dietz, M. Bathe, Nucleic Acids Res. 2012 , 40, 2862. 